\title{
Temperature reinforcement for visual discrimination training in baby chicks*
}

\author{
EVELYN LEE-TENG and STUART R. BUTLER $\dagger$ \\ Division of Biology, California Institute of Technology \\ Pasadena, California 91109
}

A fully automated training apparatus, using thermoregulation as performance incentive, was constructed to train baby chicks on visual discrimination and reversal problems. In the base condition, the $S$ is bathed in cold air in a small training cubicle. Two stimuli are back-projected onto two display windows according to a pseudorandom balanced schedule. A peck at the positive stimulus gives convective as well as radiant heat reward with adjustable temperature and duration. A peck at the negative stimulus merely advances the program to the next trial. Permanent printout records give trial-to-trial information on response choice and latency. Performance data of 64 Ss are reported.

The newly hatched chick, being a precocial bird, is an economical and convenient choice for behavioral research, but earlier studies have been hampered by the relative ineffectiveness of conventional performance incentives such as food, water, or electric shock (e.g., Dutch, 1969; Gray, Yates, Vandiver, \& Kirwan, 1967; James \& Binks, 1963). The optimal ambient temperature for chicks in the first week after hatching is about $95^{\circ} \mathrm{F}$. When maintained at lower ambient temperatures, chicks either pack closely together when in a group or give persistent distress calis when in isolation. These observations led us to consider the use of temperature reinforcement. A later report that baby chicks learned to enter the correct goalbox for radiant heat reward in a hand-training procedure (Zolman, 1969) lent further impetus to the development of an automated apparatus that dispenses convective as well as radiant heat reward for discriminative pecking. This setup can be used in a large variety of studies. In this report, some data on visual discrimination learning and its reversal will be given to illustrate the effectiveness and efficiency of the described apparatus.

\section{APPARATUS}

The Training Cubicle

The small training cubicle and its connection with the forced-air ventilation system are schematically

*Supported by U.S. Public Health Service Grant MH 03372 awarded to Professor R. W. Sperry. We thank Peter Jonkhoff and Douglas Lockwood for assistance in apparatus construction and Carolyn Kopp for assistance in data collection and analy sis.

+Now at Department of Anatomy, The Medical School, Birmingham University. Birmingham 15, U.K. presented in Fig. 1. The cubicle has inside dimensions of $5 \times 3 \times 15$ in. The four walls are made of 5/8-in. Lucite painted flat gray. The top is covered by a removable piece of $1 / 8$-in. clear glass. The cubicle is itself bottomless; when in use, it is set over several folds of paper towel on the table, so that excrement can be easily removed by change of the towels. Centered on the midline of one 3 -in. wall are two adjacent windows for stimulus presentation and response detection. They are arranged in top-bottom instead of left-right alignment so that each of the laterally situated eyes of the chick will have equal viewing of both stimuli. The division between the two windows is 9 in. from the bottom of the wall. The opposite 3-in. wall is removable and serves as a door. The platform on which the chick stands is a piece of $2 / 4-i n$. wire mesh. It can be inserted into any one of four grooves to adjust to the size of the chick. The height of the grooves ranges from $31 / 2$ to $5^{1 / 2}$ in. from the bottom of the walls. Two 1\%/4-in. air holes, centered $1 \frac{1}{2}$ in. from top and bottom of one 5-in. wall, respectively, connect the training cubicle to the ventilation system.

\section{Stimulus Display} and Response Detection

Visual stimuli are back-projected onto the two windows, each made of $1 / 2 \times 3 / 4 \times 5 / 32$ in. thick ground glass, from two miniature readout units (Industrial Electronic Engineers, Inc., Series 340). Each unit contains 12 different stimuli, including a blank, from which the $\mathbf{E}$ can select the positive stimulus and the negative stimulus by using two rotary wafer switches. Each window is hinged and lightly spring-loaded. Pecks at the window are detected photoelectrically in the following manner: A miniature Cystoscope lamp (American Cýstoscope Makers, Inc., No. 58) is mounted behind and to the left of

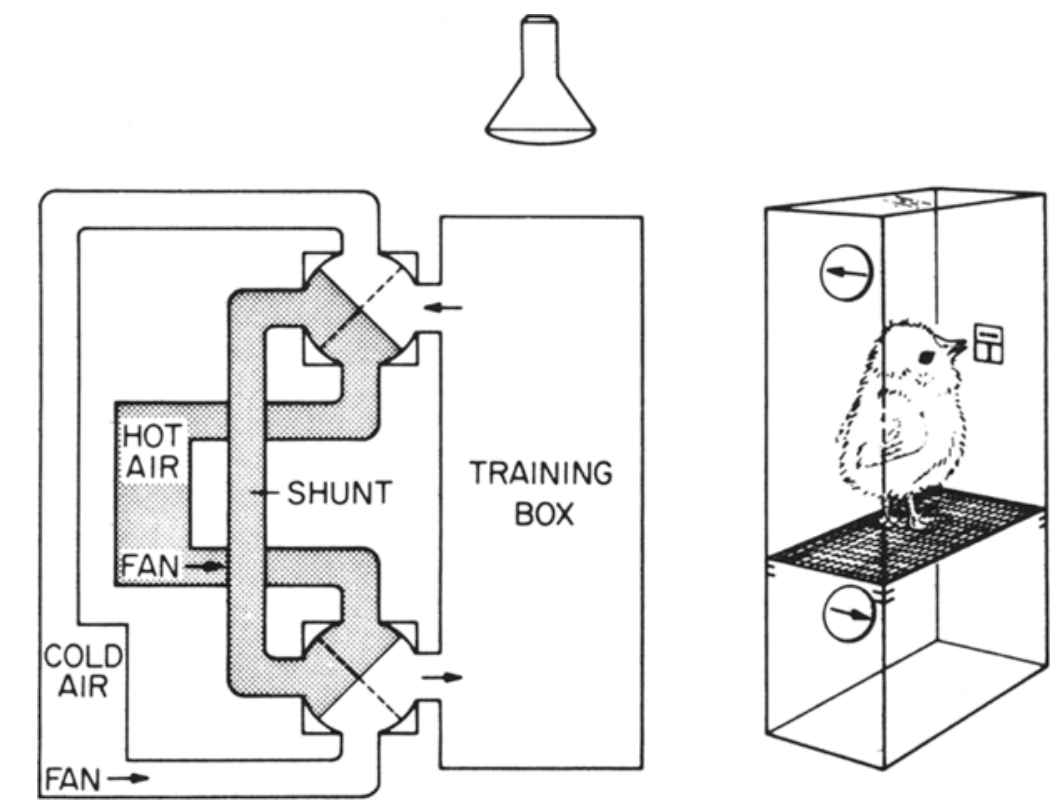

Fig. 1. Schematic presentation of the training apparatus. 


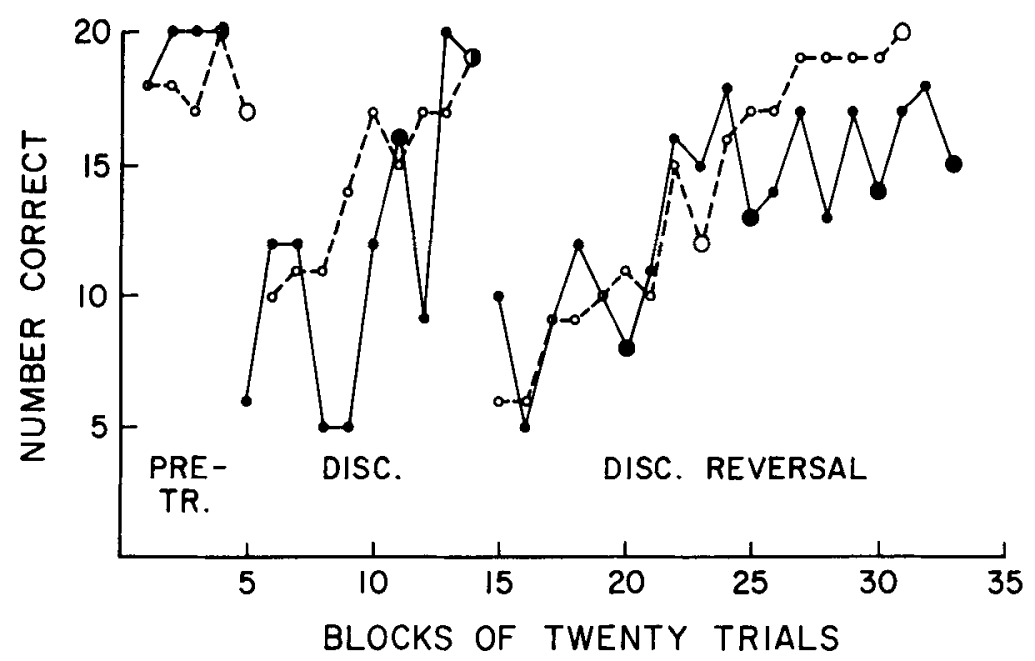

Fig. 2. Performance of two Ss. The discrimination problem for one $S$ (dashed line) was bar orientation: vertical $(S+)$ vs horizontal $(S-)$; that for the other $S$ (solid line) was colored disks: orange (S+) vs red (S-). Larger circles indicate end of sessions.

each window and a phototransistor (Texas Instruments, LS 400) to the right. The lamp shines down a brass tube ( 1 in. long, 0.09 in. i.d.) and its light is thus converted to a narrow beam. After filtering through an infrared filter fitted at the end of the tube, the now-invisible beam passes behind the window and is received by a similar brass tube, coaxial with the first and also covered by an infrared filter at its window end to eliminate reception of external visible light. The phototransistor is mounted at the far end of this second tube. The sides of the glass windows are painted black. When the chick pecks at the window, it swings back and interrupts the light beam received by the phototransistor. The output of the phototransistor therefore indicates the occurrence of a peck. In order to convert this signal to a form compatible with digital circuits automating the training apparatus, it is amplified by a field effect transistor and transformed to square wave form by a Schmitt trigger.

\section{Temperature Control}

The convective temperature control system consists of two enclosed circuits of warm and cold air, respectively. Temperature of the warm air is maintained at $97^{\circ} \mathrm{F}$ by thermoregulated electrical heater. Air in the cold circuit is chilled to $52^{\circ} \mathrm{F}$ by blowing it through a chilled-water coil connected with a cold-water bath, the temperature of which is also thermoregulated. Airflow in each circuit is forced by a fan with adjustable speed set to give 26-CFM airflow.
In the base condition, the cold air flows through the training cubicle while the warm air bypasses through a shunt duct. During the reward period, warm air is switched to flow through the training cubicle by activation of the vane in the two valves situated immediately outside the training cubicle, as shown in Fig. 1 . The valves are constructed according to a previous report (Matthews, 1969). The positions of the two vanes are controlled by two enduranceengineered 90-deg-stroke rotary solenoids (Ledox, H-2382-032). The air ducts are made of 2-in. i.d. semiinsultated flexible tubing. The volume of air in the ducts is large relative to that in the training cubicle, so that grossly different air temperatures for the chick can be achieved almost instantaneously by activation of the vanes.

A $250-W$ red bulb infrared heat lamp situated 6 in. above the training cubicle is also automatically turned on during the reward period for additional radiant heat and light.

Programming and Recording

Solid-state logic modules and accessories (BRS/Foringer) are used for programming and recording. At the start of a training session, two visual stimuli are shown on the windows, the $52^{\circ} \mathrm{F}$ cold air flows through the training cubicle, and the heat lamp is off. If $S$ pecks at the window showing the positive stimulus, the peck initiates a 5-sec reward period during which the cold air is replaced by $97^{\circ} \mathrm{F}$ warm air, the heat lamp is on, and the visual stimuli on the windows are off. If $S$ pecks at the window showing the negative stimulus, it merely initiates a 2-sec time-out during which the visual stimuli are off; the temperature condition does not change. Possible additional pecks at the windows during either the reward or the time-out period can be recorded, but do not have any effect on the rest of the programming. At the end of either the reward period or time-out, the visual stimuli come on again, and the next trial begins. The position of the positive stimulus switches between the top and the bottom windows according to a balanced pseudorandom schedule which recycles after 32 trials.

Two running counters record the total number of pecks directed to the top and the bottom window, respectively, for easy detection of possible position habit which may be eliminated by adjustment of the height of the platform. In addition, permanent printout records give trial-to-trial information as to whether or not the response is correct, as well as the intertrial intervals.

\section{BEHAVIORAL TESTS}

A total of 70 White Leghorn cockerels were used as Ss in the present series. Over a span of 2 months, they were obtained in small groups from a nearby hatchery when 1 day old. The Ss were placed in individual housing units immediately upon arrival at the laboratory at about 9:30 a.m. The room was maintained at $88^{\circ} \mathrm{F}$ and $40 \%$ humidity. Room light was on from 6:30 a.m. to $6: 30$ p.m. Each housing $u$ nit had inside dimensions of $31 / 2 \times 63 / 4 \times 7$ in. in height. The walls were made of Lucite painted flat gray. The floor and the removable top were made of wire mesh. The resemblance of the housing cubicle to the testing cubicle was designed to reduce S's fear reactions upon introduction to the latter. Food and water were supplied ad lib in small containers.

About half of the Ss started their twice-daily 20-min training sessions on Day 2. The sessions were run once each in the morning and in the afternoon, separated by $4 \mathrm{~h}$. The other Ss had their first session in the afternoon of Day 1, followed by two daily sessions from Day 2 on. Training consisted of three successive stages: pretraining, discrimination training, and discrimination reversal. The purpose of pretraining was to teach the $S$ that pecking at either window might result in heat reward. The positive stimulus was a 1/16-in. black dot centered on a 3/8-in. illuminated circle. The negative stimulus was simply a blank, with no back-projected stimulus on the 'window. For 
discrimination training, the $\mathrm{Ss}$ were divided into four groups. Groups A and $B$ were trained to discriminate between a red and an orange disk, each color being the positive stimulus for one group. Groups $\mathrm{C}$ and D were trained to discriminate between a horizontal and a vertical bar, each orientation being the positive stimulus for one group. In discrimination reversal, the previously positive stimulus became negative, and vice versa. Records were examined only at the end of each training session. If a $S$ had reached the criterion of 12 consecutive correct responses, it was put on the next stage of training starting the following session.

Of the $70 \mathrm{Ss}$, two were excluded because of procedural errors. Four more were eliminated since they failed to complete training within 14 sessions because of a position habit or a very low pecking rate. The resulting $64 \mathrm{Ss}$ were divided evenly among the four groups. All groups had the same pretraining; about half of the Ss in each group started their training in the afternoon of Day 1 , while the others started in the morning of Day 2. Results show that the Day 1 session was of little use because most Ss gave few or no responses; all Ss reached criterion on pretraining at about the same time, whether they had had the Day 1 session or not. Of the 29 Ss who started pretraining on Day 1 , the mean value for the number of sessions to criterion (including the session during which criterion was reached) was 2.38 , and the SD was 1.00. The corresponding values for the 35 Ss who started training on Day 2 were
1.26 and 0.55 . The differences between the two groups were highly significant for both the means (C.R. $=5.33, p<.001)$ and the SDs (C.R. $=3.00, p<.01$ ). Since the added Day 1 session not only failed to speed up learning, but also resulted in more variability, as reflected in the larger SD, subsequent studies in our laboratory have eliminated the Day 1 session altogether.

An analysis of variance was performed on the session-to-criterion scores according to group membership and stages of training. The results show that the four discrimination problems were comparable in difficulty, but discrimination reversal was more difficult than the initial discrimination training $[F(3,60)<1$ for the four groups; $F(2,120)=48$, $\mathrm{p}<.001$ for the three stages; $F(6,120)<1$ for the Group by Stage interaction ]. For the four groups thus combined, the mean number of sessions to criterion was 1.77 for pretraining, 1.78 for discrimination and 2.41 for reversal; the corresponding SDs were $0.96,0.86$, and 1.06, respectively. All $64 \mathrm{Ss}$ completed the three stages of training by 7 days of age; 50 of them did so when only 3 or 4 days old.

Trial-to-criterion and error-to-criterion scores, together with more detailed data analysis, will be incorporated in a separate report (Lee-Teng, in preparation). As an example, performances of two Ss are presented in Fig. 2, showing number of correct trials in 20-trial blocks in consecutive sessions and stages of training. Since the number of trials in each session is determined by the S's frequency of response, the sessions usually do not end with an even 20-trial block. For simplicity, the following rule was adopted in data plotting: if the last block of a session had less than 10 trials, it was ignored in plotting; if it had more than 10 trials, the proportionate correct number for 20 trials was plotted.

Although most experiments on thermo-reinforcement used a heat lamp for reward, warm air was added in our studies to provide better temperature control and a more comfortable reward condition for the Ss. Other advantages of the convective air systems have been elaborated elsewhere (Matthews, 1969). In a separate experiment (Lee-Teng, in preparation), the combined use of both radiant and convective heat reward has been found to be more effective than using either alone as reinforcement for pecking in baby chicks.

\section{REFERENCES}

DUTCH, J. A runway for chicks. Behavior Research Methods \& Instrumentation, $1969,1,189-190$.

GRAY, P. H., YATES, A. T., VANDIVER, D. R., \& KIRWAN, K. A reverse Kamin effect in the escape and avoidance conditioning of newly hatched chicks. Psychonomic Science, 1967, 9, 507-508.

JAMES, H., \& BINKS, C. Escape and avoidance learning in newly hatched domestic chicks. Science, 1963, 139, 1293-1294.

MATTHEWS, T. J. A convective thermal controller for behavioral experiments. Behavior Research Methods Instrumentation, 1969, 1, 126-128.

ZOLMAN, J. F. Stimulus preferences and form discrimination learning in young chicks. Psychological Record, 1969, 19, 407-416. 